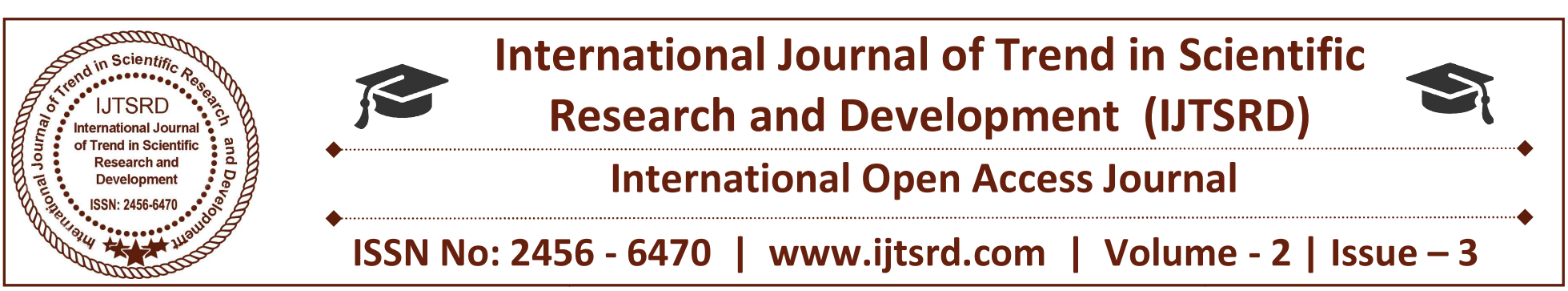

\title{
An Adaptive IoT Framework: using FPGA Based SOC for varying Applications
}

\author{
Komal Gophane, Dr. P. C. Bhaskar \\ Department of Technology, Shivaji University, \\ Kolhapur, Maharashtra, India
}

\begin{abstract}
With the evolution of IoT, it is expected to create large impact on human lives. IoT is a region where world convergences with physical world. The IoT is an intelligent network which connects all things to the Internet for the purpose of exchanging information and communicating through the information sensing devices. It achieves the goal of intelligent identifying, locating, tracking, monitoring, and managing things. IoT (Internet of Things) is a system where objects are embedded with sensor technology to interact with each other over wireless communication medium to generate exchange and transfer data without human interaction. In this paper we presented, FPGA based Adaptive IoT framework for Distinct Applications, based on Cloud server. In this, the controllers can read, collect, and transmit huge volume of data over the internet. This paper is focusing, the development of an adaptive IoT system that can be used in any field for wireless communication.
\end{abstract}

Keywords: IoT(Internet of Things), Spartan 3 FPGA, Raspberry pi3, cloud server, sensors.

\section{INTRODUCTION}

The keyword Internet of Things was first introduced in 1999 by Kevin Ashton for Supply Chain Management.

\section{A. Definition of Internet of Things:}

"The Internet of Things (IoT) is the network of physical devices, vehicles, buildings and other items- embedded with electronics,

software, sensors and network connectivity that enables these objects to collect and exchange"

\section{B. Need of Internet of Things:}

IoT becomes more and more popular day by day to maximum use of Broadband Internet as the internet users increases. Using IoT, connection cost can be decreased by Wi-Fi connections, built-in Sensors in devices and maximum number of devices connects together by a Common medium.[15]

The "Internet of Things" states to a global network of objects, or "things," seamlessly connected to the internet which can basically shift the way we interact with our surroundings. This internet of things allows physical objects to see, listen, think, and perform tasks by sharing information and coordinating decisions. IoT transforms objects from being traditional to being smart by using its underlying technologies such as sensor networks, embedded devices, communication technologies, and ubiquitous and pervasive computing. The Number of physical objects connected to the internet at an unprecedented rate, leading to diverse the range of applications including but not limited to smart greenhouse [11], remote healthcare [8], agriculture [17], smart cities [14] and smart water management [12].

It is expected by 2020 around 30 billion of smart devices installed and connected to the cloud, representing four devices for each individual person in the world [18]. In the IoT model, many objects surrounding us will be connected into networks in one 
form or another. Internet of things (IoT) is a smart technology that connects anything, anywhere, at any time [21]. Internet of Things ${ }^{e e}$ (IoT) is used to refer to many concepts related to the extension of the Internet and the Web into the physical daily life. This is supported by means of the extension of distributed devices with embedded identification, sensing and/or actuation capabilities. IoT envisions a future in which both digital and physical entities can be linked, by means of appropriate information and communication technologies, to enable a big range of applications and services [17]. The proposed system is FPGA based IoT framework. It consists of an IoT based prototype with sensors to measure physical data, with facility to transmit information over the Cloud. The literature reviews are done for performing an efficient comparison of different ways for collection of physical data: Traditional Methods. The rest of the paper is organized into the following sections: Literature reviews are covered in section 2. Section 3 covers proposed work. Finally, concluding remarks are given in section 4 .

\section{RELATED WORK}

A brief survey has been done regarding IoT system and its applications. Following paragraphs summarizes literature survey.

\section{Health monitoring using IoT and RFID:}

SarfrazFayazKhan[2017][4] proposed a complete continuous monitoring and smart healthcare monitoring system designed by using the IoT and RFID. In this system, the different sensors are fixed in the patient body and according to the signals from sensors, RFID and IoT the patient can be monitor. The signals generated from these sensors transmitted to the microcontroller. Then microcontroller forwards this sensor data to RFID/Bluetooth devices. RFID/Bluetooth devices wirelessly transmit these signals to the mobile phone for the transmission of data through internet to the specific destination. All these operations can be done into four different layers- sensor layer, Network layer, Internet layer, Service layer.

Vehicle traffic congestion reduction system using IoT: Mohammad Reza Jabbarpour*, Armin Nabaei and HoumanZarrabi[2016][9]proposed a Vehicle Traffic congestion Reduction System using IoT(Internet of Things). Traffic jam conditions at pick hours in large cities causes one direction of highway is congested while there are a few vehicles on the other direction. This congestion condition can be reduce using Intelligent Guardrails (IGs).An IoT applications, Intelligent Guardrails ${ }^{e e}(\mathrm{IG})$ is presented which apply vehicular networks to detect traffic condition of the roads and uses electronic and mechanical techniques for increasing the capacity(number of lanes) of congested side of highway by decreasing the non-congested side of it.

\section{IoT: Challenges and Applications}

Khaled Abdulla Al Rabaiei, Saad Harous [2016][17] IoT envisions a future in which both digital and physical entities can be linked, by means of appropriate information and communication technologies to enable a big range of applications and services. In this paper, different applications of IoT are collected, classified and elaborated into three domains: Society, Industry, and Environment. Some consumer-ready IoT platforms have been mentioned. Then some of the most important Limitations facing the spread of the IoT applications were discussed.

\section{Industrial Treats securing using IoT}

Muhammad Usman, NazarAbbas [2014][30] proposed the Industrial Treats securing using IoT. This security based on IoT and on any doubtful act alert the authorized person of a corporate center by calling or messaging at his/her cell phone and the surroundingees by alarm simultaneously. The nine sensors are connected to the arduino microcontroller. A cell phone is also connected to the system. Arduino microcontroller takes input from these nine sensors and if doubtful is detected by the sensors, the arduino give alert on cell phone. This system can helps people to protect their homes, offices, banks or any corporate center at less cost.

\section{PROPOSED WORK}

During recent years, a pragmatic term Internet of Things (IoT) has attracted global interest with the speculation that 50 billion devices would be connected to the Internet by 2020 for machine-tomachine and man- to-machine communications. IoT may even lead to a new industrial revolution. IoT is already here and in use. Nowadays IoT represents a collection of billions of tiny smart connected devices and sending secured data through the internet to dedicated online cloud servers. Internet of Things (IoT) short-range, ultra-low power communication 
technologies comprises one of the most rapidly evolving research areas attracting significant interest. From a complex Smart City Monitoring System to a simple Smart Street Lamp or from an advanced security system to a Patient Vital Signs Monitoring System, all these "Things" have a common basic requirement: connectivity. [18]

The way the IoT devices implement their selected technology will affect the application area and thus, the device ${ }^{e e}$ s interoperability and connectivity. The main focus of proposed work will go into the Communication Model since the connectivity and the interoperability plays a key role on the IoT devices. This work will go beyond state-of-the art presenting a FPGA-based solution for the IoT. The proposed work will focus on the connectivity and the interoperability of the edge-devices, by implementing an IoT protocol stack on cost-effective SoC FPGA platform with an integrated hardcore processor. This solution aims to increase the performance and thus improve the efficiency of the edge-devices by implementing design choices, mainly by offloading critical software features (OS or protocol stack) to hardware. This approach aims to evaluate, as a proof- of-concept, future efficient $\mathrm{SoC}$ implementations with the new improvements and added features. As technology moves forward, the customizable FPGA- IoT platform is essential. There is no "One-size-fits- all" solution. So, design a FPGA based adaptive framework for distinct applications is needed.

\subsection{System Block Diagram:}

The proposed methodology comprises an architecture that is subdivided into different systems (layers). The different layers in the proposed system include sensors, microprocessor, a wireless transceiver, gateway, and application management cloud server.

The proposed methodology requires study of various applications from different context for IoT. Then the study of IoT architecture reference model. Use different sensors and the controller and the internet, in this proposed work. Then finally design and develop FPGA architecture based adaptive IoT framework for various applications.

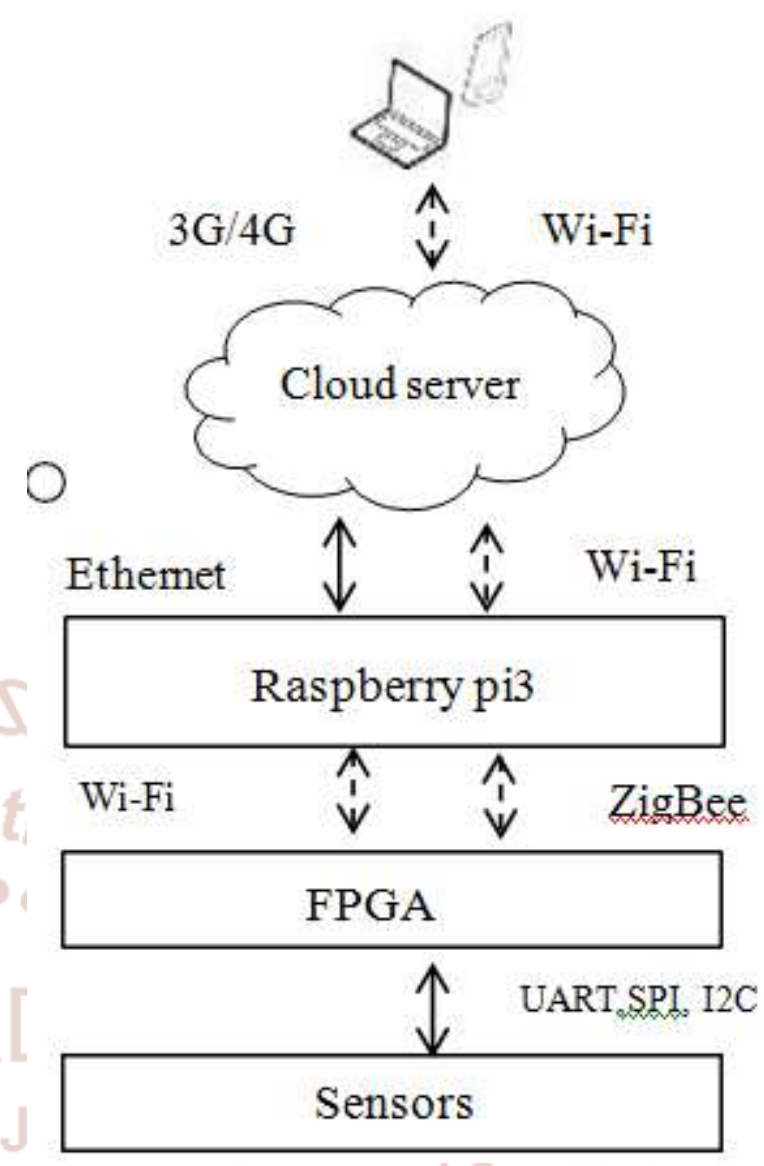

Fig 3.1 Proposed system block diagram

The Fig3.1 shows a block diagram of the proposed system. The system contains sensors, FPGA, ARM controller, cloud server, transceivers. Aiming to improve the performance of the IoT communication stack, FPGA-Based IoT architecture is proposed. It is based on a Smart fusion which is mainly composed of an ARM processor and the FPGA for specific hardware implementations.

\section{SYSTEM IMPLEMENTATION}

For system implementation we required hardware and software tools. The physical layer of the proposed system consists of the different application specific sensors, as used to measure the physical parameters. Sensors example, Healthcare sensor (electrocardiogram, oximeter, heart rate, ,ADXL), building ambient sensors(temperature, humidity, light intensity, presence, and indoor positioning), structural health sensors(piezoelectric, and acoustic), Agriculture sensors(soil moisture, soil temperature, wind speed, wind direction, solar radiation and leaf wetness), home sensors(PIR, LDR,), Industry sensors(Smoke sensor, IR). Sensing and data forwarding rates and wireless technology used have a strong influence on energy consumption. Sensors are 
analog or digital. The analog sensors need to connect to ADC (Analog to digital converter) for getting digital form of sensed data. Based on performance improvement requirement, low-power microcontroller with few inputs could be replaced by high performance microcontroller with multiple input lines. We are chosen FPGA Spartan 3for sensor connectivity. FPGA Spartan 3 family has better capacity of Internal RAM, the total number of I/Os and improved clock management function FPGA having Up to $633 \mathrm{I} / \mathrm{O}$ pins and $622+\mathrm{Mb} / \mathrm{s}$ data transfer rate per I/O and parallel processing. We have selected RASPBERRY pi3 as Internet Gateway for cloud connectivity. Raspberry pi 3 is the third generation Raspberry pi which is a powerful credit card sized single board computer can be used for IoT applications, Robotics, server/cloud server, Low cost pc/tablet/laptop. Cloud server is responsible for facilitating the end users ability to access the sensed data. This is achieved by implanting several services including, but not limited to, data storage, data analysis, and data visualization.

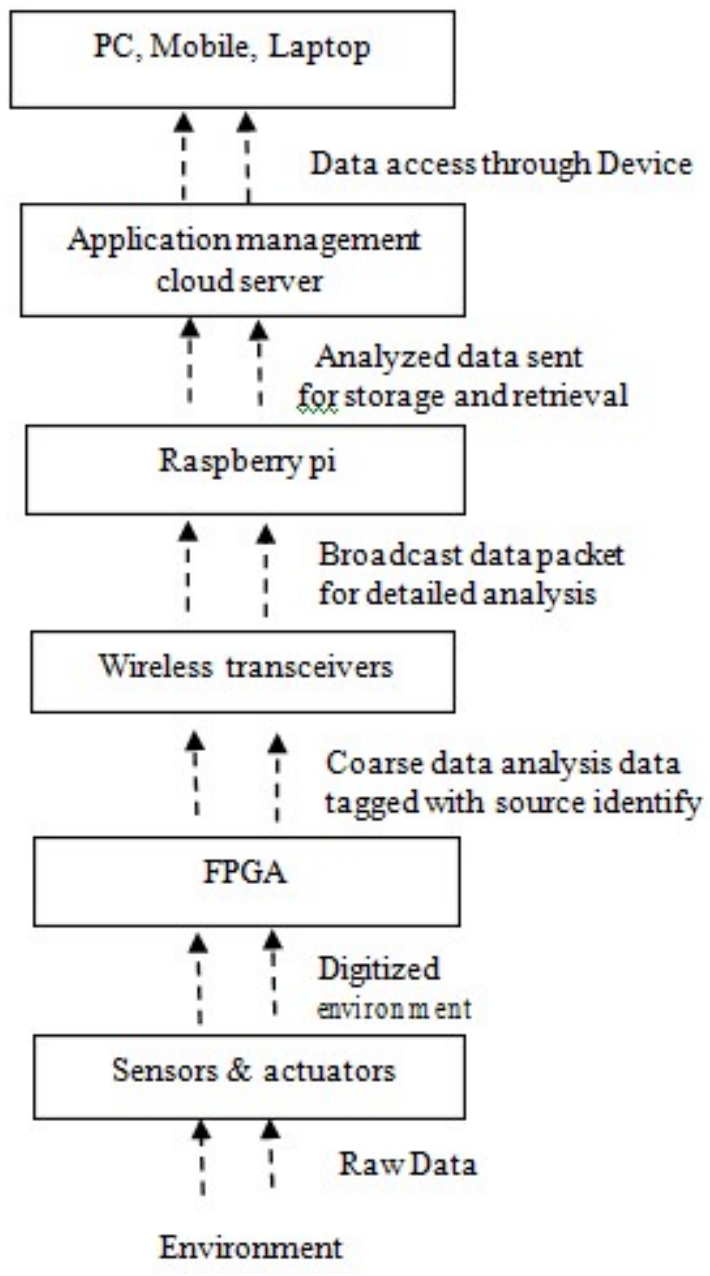

Fig 3.2 Information flow diagram
The Fig.3.2 show information flow diagram for proposed system and Fig. 3.3 show the proposed FPGA based adaptive IoT framework for distinct applications.

\section{CONCLUSION}

The FPGA based Adaptive IoT(Internet of Things)Framework which is a single system can be used for different applications, but not limited to health monitoring, Home monitoring, Agriculture monitoring, Industrial monitoring. The things here are the sensors, which are controlled by FPGA spartan3.Information from sensors are send to cloud server where the data storage, manage and operation. There is no need of hardware storage system for information storage due to use of cloud server. This is low cost \& user friendly system. India Digitization is very easy by using this Adaptive IoT framework.

In the future work, We would like to develop this proposed adaptive IoT framework system which can use in different applications and to make use of this system where IoT not previously possible.

\section{REFERENCES}

1. Laith Farhan, Sinam T. Shukar, Ali E. Alissa, "A survey on the Challenges and Opportunitiess of the Internet of Things(IoT)", 2017 Eleventh International conference on Sensing Technology(ICST),2017

2. - M. 7 Radovan*, B.Golub"Trends in IoT Security"MIPRO 2017, Opatija, Croatia, May 2226, 2017

3. Gopal Krishna Shyam, Sunilkumar S. Manvi,PriyankaBharathi "Smart Waste Management using Internet-of -Things" 2017 second International Conference on Computing and Communication Technology (ICCCT ${ }^{\mathrm{ee}} 17$ ) 2017 IEEE , pp.199-203

4. SarfrazFayaz Khan "Health Care Monitoring System in Internet of Things (loT) by Using RFID" 2017 the 6th International Conference on Industrial Technology and Management(ICITM) 2017 IEEE, pp.198-204

5. Yongbin Zhao, Ning Cao "Research on Traceability of Agricultural Products Based on Internet of Things" 2017 IEEE International Conference on Computational Science and Engineering (CSE) and IEEE International 
International Journal of Trend in Scientific Research and Development (IJTSRD) ISSN: 2456-6470

Conference on Embedded and Ubiquitous Computing (EUC) 2017 IEEE, Vol.4, pp.414-417.

6. Bilal Javed, MianWaseem Iqbal1\&Haider Abbas "Internet of Things (IoT) Design Considerations for Developers and Manufacturers" 5th IEEE International Workshop on Smart Communication Protocols and Algorithms (SCPA 2017) 2017 IEEE, pp.834-839.

7. Feng Zhang, Min Liu, Zhuo Zhou, WeimingShen "An IoT Based Online Monitoring System for Continuous Steel Casting” IEEE INTERNET OF THINGS JOURNAL, 2016 IEEE, Vol.3, Iss.6, pp.1355- 1363 .

8. Vikas Vippalapalli "Internet of things (IoT) based smart health care system" 2016 International conference on Signal Processing, Communication, Power and Embedded System (SCOPES) 2016 IEEE, pp.1229- 1233.

9. Mohammad Reza Jabbarpour*, Armin Nabaei and HoumanZarrabi "Intelligent Guardrails: An IoT Application for Vehicle Traffic Congestion Reduction in Smart City" 2016 IEEE International Conference on Internet of Things (iThings) and IEEE Green Computing and Communications (GreenCom) and IEEE Cyber, Physical and Social Computing (CPSCom) and IEEE Smart Data (SmartData) 2016 IEEE, pp.7-13.

10. AyushKapoor, Suchetha I Bhat, SushilaShidnal, AkshayMehra "IMPLEMENTATION OF loT (INTERNET OF THINGS) AND IMAGE PROCESSING IN SMART AGRICULTURE" 2016 International Conference on Computational Systems and Information Systems for Sustainable Solutions(CSITSS),2016 IEEE, pp.21-26.

11. Aarti, 2Pooja Mittal "REVIEW PAPER ON HOME AUTOMATION USING INTERNET OF THINGS" International Journal of Emerging Trends \& Technology in Computer Science (IJETTCS) Volume 5, Issue 4, July - August 2016

12. Nyoman Putra Sastra,Dewa Made Wiharta "Environmental Monitoring as an IoT Application in Building Smart Campus of UniversitasUdayana" $2016 \quad$ International Conference on Smart Green Technology in Electrical and Information system (ICSGTEIS), 2016 IEEE, pp.85-88.

13. Francisco Javier Ferrández-Pastor, Juan Manuel García-Chamizo, Mario Nieto-Hidalgo, Jerónimo Mora-Pascual and José Mora-Martínez
"Developing Ubiquitous Sensor Network Platform Using Internet of Things: Application in Precision Agriculture” Article, Sensors 2016.

14. Sylvain Kubler, Je're'my Robert, Ahmed Hefnawy, KaryFrämling, Chantal Cherifi, and AbdelazizBouras "Open IoT Ecosystem for Sporting Event Management" JOURNAL OF LATEX CLASS FILES, VOL. 14, NO. 8, AUGUST 2015. IEEE Access, Vol.5, pp.70647079.

15. Talwana Jonathan Charity, Huang JianHua "Smart World of Internet of Things (IoT) and It's Security Concerns" 2016 IEEE International Conference on Internet of Things (iThings) and IEEE Green Computing and Communications (GreenCom) and IEEE Cyber, Physical and Social Computing (CPSCom) and IEEE Smart Data (SmartData) 2016 IEEE, pp.240-245.

16. HongmingCai, Senior Member, IEEE, BoyiXu, Member, IEEE, Lihong Jiang, Member,IEEE, and Athanasios V. Vasilakos, Senior Member, IEEE,'IoT- based Big Data Storage Systems in Cloud Computing: Perspectives and Challenges", IEEE Internet of Things Journal Citation information: 10.1109/JIOT.2016.2619369,2016

17. Khaled Abdulla Al Rabaiei, SaadHarous"Internet of Things: Applications and Challenges" 2016 12th International Conference on Innovations in Informatio Technology (IIT)978-15090-5343-8/16/\$31.00 (C)2016 IEEE

18. T. Gomes, F. Salgado, S. Pinto, J. Cabral and A. Tavares Centro Algoritmi - University of Minho"Towards an FPGA-Based Network Layer Filter for the Internet of Things Edge Devices"978-1-5090- 1314-2/16/\$31.00 (C2016 IEEE

19. VikasVippalapalli,Snigdha Ananthula2 "Internet of things (IoT) based smart health care system"International conference on Signal Processing, Communication, Power and Embedded System (SCOPES)-2016,978-1-50904620-1/16/\$31.00 (2016 IEEE

20. Francisco Javier Ferrández-Pastor *, Juan Manuel García-Chamizo, Mario Nieto-Hidalgo, Jerónimo Mora-Pascual and José MoraMartínez’Developing Ubiquitous Sensor Network Platform Using Internet of Things: Application in Precision 
International Journal of Trend in Scientific Research and Development (IJTSRD) ISSN: 2456-6470

Agriculture", Sensors 2016, 16,1141; doi:10.3390/s16071141,2016www.mdpi.com/jour nal/sensors.

21. NavroopKaur and SandeepK. Sood"An EnergyEfficient Architecture for the Internet of Things (IoT)" IEEE SYSTEMS JOURNAL 1932-8184 @ 2015 IEEE

22. Yunchuan Sun, Member, IEEE, Houbing Song, Senior Member, IEEE, Antonio J. Jara,Member, IEEE, and RongfangBie, Member, IEEE "Internet of Things and Big Data Analytics for Smart and Connected Communities "IEEE ACCESS, VOL. 14, NO. 8,AUGUST 2015,2169-3536 (c) 2015 IEEE

23. T. Gomes, J. Brito, H. Abreu, H. Gomes and J. Cabral" GreenMon: An Efficie Wireless Sensor Network Monitoring Solution for Greenhouses",978-1-4799-7800-7/15/\$31.00 (C)2015 IEEE

24. S. Anjana, M. N. Sahana, S.Ankith, K. Natarajan, K. R. Shobha, and A. Paventhan, "An IoT based 6LoWPAN enabled experiment for water management," in Proceedings of the 9th IEEEInternational Conference on Advanced Networks and Telecommuncations Systems (ANTS "15), Kolkata, India, December 2015.

25. CHUNSHENG ZHU1, (Student Member, IEEE), VICTOR C. M. LEUNG1, (Fellow, IEEE), LEI SHU2, (Member, IEEE), AND EDITH C.-H. NGAI3, (Senior Member,IEEE)"Green Internet of Things for Smart World" VOLUME 3, 2015,2169- 35362015 IEEE
26. T. Gomes, J. Brito, H. Abreu, H. Gomes and J. Cabral "GreenMon: An Efficient Wireless Sensor Network Monitoring Solution for Greenhouses" 2015IEEE International Conference on Industrial Technology(ICIT) 2015 IEEE, pp.2192-2197.

27. M. Nakamura and L. Du Bousquet, "Constructing execution and life-cycle models for smart city services with self-aware IoT," 2015 IEEE 12th IEEE International Conference onAutonomic Computing(ICAC " 15 ), pp. 289-294, July 2015.

28. Klara Nahrstedt"Internet of Mobile Things: Challenges and Opportunities"PACT'14,Edmonton, AB, Canada. ACM 978-1-4503-28098/14/08.http://dx.doi.org/10.1145/2628071.26359 31, August 24-27, 2014.

29. Shanzhi Chen, Senior Member, IEEE, HuiXu, DakeLiu,Senior Member, IEEE, Bo $\mathrm{Hu}$, and Hucheng Wang"A Vision of IoT: Applications, Challenges, and Opportunities With China Perspective"IEEE INTERNET OF THINGS JOURNAL, VOL. 1, NO. 4, AUGUST 2014.

30. Muhammad Usman, Nazar Abbas "On the Application of IOT (Internet of Things) for Securing Industrial Threats" 2014 12th International Conference on Frontiers of Information Technology, 2014 IEEE, pp.37-40.

31. Sean Dieter Tebje Kelly, Nagender Kumar Suryadevara, and Subhas Chandra Mukhopadhyay "Towards the Implementation of IoT for Environment Condition Monitoring in homes" IEEE SENSORS JOURNAL,2013 IEEE, VOL. 13 , Iss. 10, pp.3846- 3853 . 